\title{
Two-hit Hypothesis and Multiple Organ Dysfunction Syndrome
}

\author{
Butt I, ${ }^{1}$ Shrestha BM ${ }^{1}$ \\ Sheffield Kidney Institute, Sheffield, UK
}

\section{ABSTRACT}

A severe insult in the form of infection or trauma primes the host immune system so that a subsequent, relatively trivial insult produces a markedly exaggerated host immune response, which can lead to multiple organ dysfunction syndrome (MODS) and death. This forms the basis of the "two-hit hypothesis" (THH), which is being increasingly recognised as an important cause of morbidity and mortality following severe injury and sepsis, particularly in intensive care settings. Appreciation of the impact of repeated insults and pathophysiology of MODS is vital in the prevention of this serious complication. We describe a case which illustrates the concept of THH and MODS and present a review of literature on this subject.

Key words: laparotomy, multiple organ dysfunction, sepsis, two-hit hypothesis

\section{INTRODUCTION}

Patients exposed to a severe primary insult, e.g. infection or trauma, can develop rapidly progressive multiple organ dysfunction syndrome (MODS) if a subsequent immune stimulus, "second hit", occurs. The initial serious injury primes the immune system for an exaggerated inflammatory response to a subsequent stimulus such as nosocomial infection or surgical trauma, which has promulgated the concept of "twohit hypothesis" (THH). ${ }^{2}$ At present, MODS remains the most common cause of death in the surgical intensive care units (ICU). We present a case which illustrates the concept of the THH and MODS and review recent literature on this topic.

\section{CASE REPORT}

An 86-year-old female was admitted, via accident and emergency department, with three days, history of lower abdominal pain, vomiting and absolute constipation. In the past, she had undergone hysterectomy 30 years ago for dysfunctional uterine bleeding. She had no significant cardio-respiratory co-morbidities. On examination, she was alert and hemodynamically stable. The abdomen was distended with mild lower abdominal tenderness without any evidence of peritonitis. Routine blood results showed normal hemograms, renal and liver function tests. Plain abdominal X-ray (Figure 1) showed dilated loops of small intestine. Chest X-ray (Figure 2) revealed consolidation of both lung bases with no evidence of pneumo-peritoneum. As the abdominal pain worsened, a CT scan of the abdomen was performed which showed features of small intestinal obstruction. At laparotomy, the ileum was grossly distended and adherent to the old midline wound scar with rotation on its mesentery. However, the blood supply to the segment of intestine was well preserved. Several interloop adhesions were taken down and mass closure of the abdominal wound was performed with loop 0 PDS.

\footnotetext{
Correspondence:

Dr. B.M. Shrestha

Sheffield Kidney Institute

Herries Road, Sheffield, S5 7AU, UK

Email: shresthabm@doctors.net.uk
} 
Butt et al. Two-hit Hypothesis and Multiple Organ Dysfunction Syndrome

On the second post-operative day, the patient was agitated and confused and gradually became oliguric. The abdomen was tender, grossly distended with absent bowel sounds. There was reduced air entry at both lung bases. The white blood cell count had fallen to $1.6 \mathrm{x}$ $10^{9} / \mathrm{L}$ from $5.2 \times 10^{9} / \mathrm{L}$ at the time of admission. Repeat contrast-enhanced CT scan showed massive bilateral pleural effusion and free fluid in the peritoneal cavity (Figure 3). The loops of small intestine were grossly dilated but without any evidence of ischaemia as there was normal enhancement. Resuscitation was carried out with intra-venous infusions and continuous positive airway pressure ventilation which restored her urine output and mental status. At this stage, she did not require any inotropic support to maintain haemodynamic stability.

At second-look laparotomy, there was a litre of bile in the peritoneal cavity. The small intestine, although dilated, was normal in appearance. Careful search revealed a $4 \mathrm{~mm}$ perforation over the fundus of the gall bladder through which bile was leaking. Cholecystectomy was carried out and the abdomen was closed following a thorough peritoneal lavage with saline. In the immediate post-operative period, she required continuous infusion of noradrenaline and dobutamine to maintain the blood pressure. She remained oliguric with severe metabolic acidosis (blood $\mathrm{pH}$ of 7.3, base deficit of 6.2 and

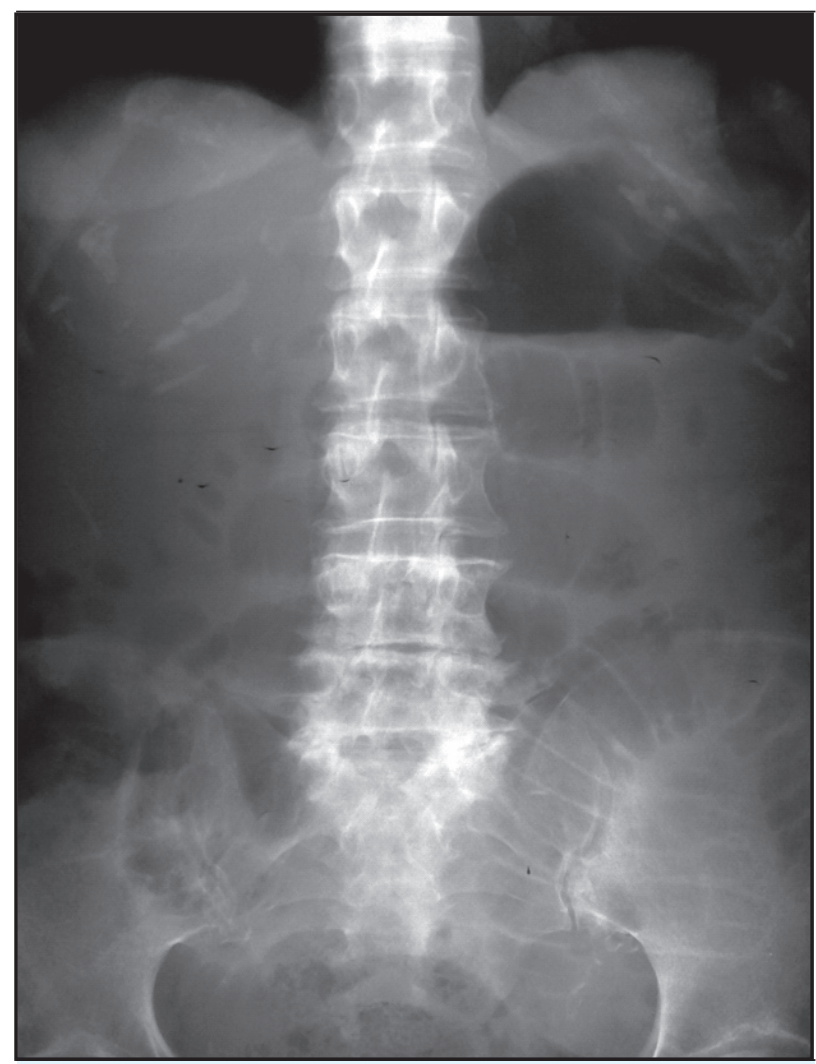

Figure 1. Abdominal $\mathbf{x}$-ray showing small intestinal obstruction blood lactate of $5 \mathrm{mmol} / \mathrm{L}$ ). Despite intensive care management, progressive deterioration led to death after 18 hours following the second-look laparotomy.

\section{DISCUSSION}

The origin of the concept of THH lies in clinical observations made over 20 years ago. The development of THH after a second surgical procedure, e.g. fracture fixation in an otherwise young fit patient who had undergone splenectomy and seemed to be improving from the initial insult, stimulated the research promulgating the concept of $\mathrm{THH}^{3,4}$ Fundamental to the concept of the $\mathrm{THH}$ is a pathologically exaggerated inflammatory response to a second physiological insult. The exaggerated nature of this response is a consequence of the 'priming' of immune effector cells by the first insult. ${ }^{5}$ Cell priming is defined as an enhanced response to a stimulus as a consequence of prior stimulation by another agonist. If the second insult occurs during the window of vulnerability prior to down-regulation of the primed immune reactants, the

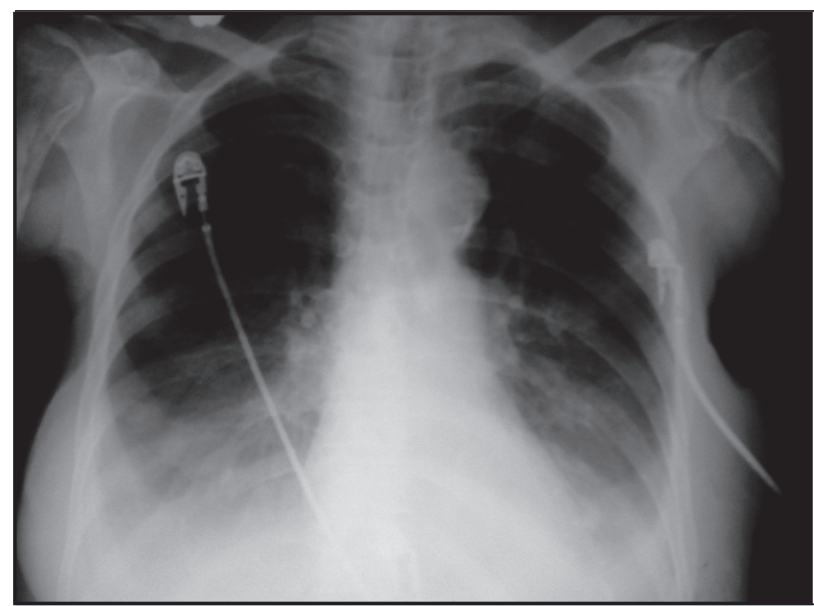

Figure 2. Chest x-ray showing bilateral basal consolidation

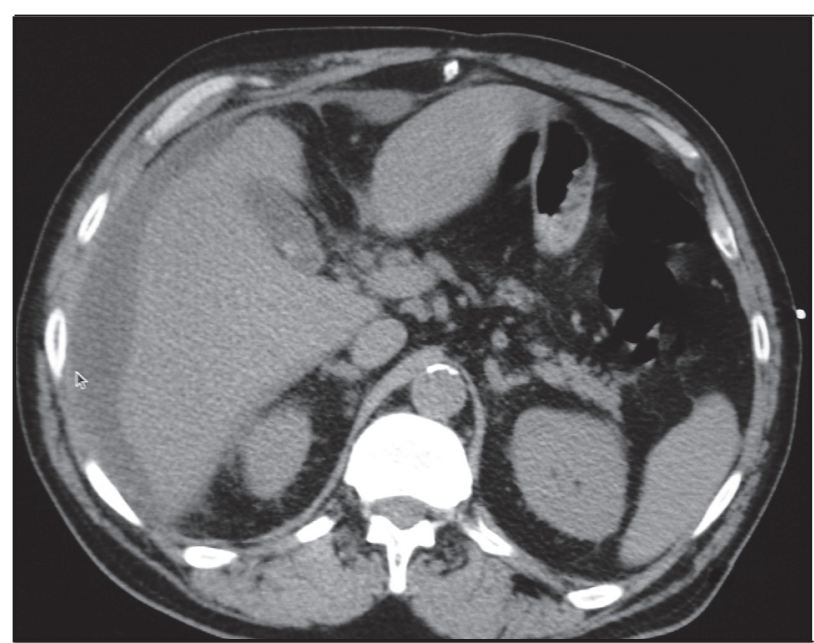

Figure 3. Computerised tomographic scan showing free intraperitoneal fluid

JNMA || VOL 47 | NO. 2 | ISSUE 170 || APR-JUN, 2008 
Butt et al. Two-hit Hypothesis and Multiple Organ Dysfunction Syndrome

inflammatory response is seen to balloon beyond that which would otherwise have occurred. ${ }^{6}$ In our case, the systemic response to biliary peritonitis and second laparotomy, having been primed by intervention 48 hours earlier, proved terminal.

The response of the polymorphonuclear neutrophils (PMN) to priming is well characterised. ${ }^{7}$ Generation of free radicals like reactive oxygen species (ROS), and reactive nitrogen species (RNS), degranulation of enzymes, cytokine expression, integrin expression and delayed apoptosis, amongst other responses, has shown to be enhanced after priming with agents such as tumour necrosis factor (TNF)-alpha interleukin-6 (IL6), IL-8, ILK-10, platelets activating factor (PAF), and lipopolysaccharides (LPS). ${ }^{8,9}$ Studies have shown that monocytes and macrophages may also become similarly primed after a severe insult. Activated endothelium, with increased expression of adhesion molecules, intercellular adhesion molecules (ICAM) and vascular cell adhesion molecules (VCAM) have a central role in the magnified response to the second hit. ${ }^{10}$

The understanding of the pathophysiology and management of MODS has progressively evolved since the establishment of the first ICU in Baltimore, USA in the 1950s. ${ }^{11}$ Baue in 1975 introduced the concept of MODS and suggested that each organ abnormality was simply the local manifestation of a systemic process, hence, therapy should be targeted to the fundamental mechanism in its pathogenesis. ${ }^{12}$ Due to the complex nature of the disease process and limitations in the ability to describe and characterise them, the therapy for prevention of MODS still remains in infancy.

MODS is preceded by the activation of adaptive host stress response to a wide variety of systemic insults including surgery, trauma, burns and severe infections, and includes changes in the cardiorespiratory function, increased microvascular permeability, activation of innate immune mechanisms, and alteration in the intermediary metabolism. This is termed sepsis when caused by infection and systemic inflammatory response syndrome when considered independent of the cause. ${ }^{13}$ The adaptive response to these injurious agents can precipitate cellular and organ dysfunction, which may ultimately lead to death of the host. Recovery from MODS may result in partial or total permanent loss of functions of vital organs as a consequence of cellular damage and fibrosis that occur during the process of recovery. ${ }^{14}$

Intra-abdominal pressure (IAP) of over $25 \mathrm{~mm} \mathrm{Hg}$ leading to abdominal compartment syndrome (ACS) is often associated with MODS. ${ }^{15}$ It is a common practice to measure IAP regularly in intensive care settings to recognise ACS early so that measures such as nasogastric or colonoscopic decompression of the intestines, laparostomy with wound management using Bagota bag, Wound Manager $^{\circledR}$ or vacuumassisted closure devices are employed to reduce the mortality. ${ }^{16}$

Prevention of MODS should be in the highest priority in all clinical settings, which is possible through avoidance of secondary insults. Halting the progression of existing organ dysfunction while preventing the development of new organ dysfunction should be approached through optimisation of haemodynamic, metabolic, and immune homeostasis. Occult infection, particularly infection arising within the abdomen, is an important risk factor for MODS, which should be carefully looked for in all cases of unexplained MODS. ${ }^{18}$ Patients with established MODS should be managed in a high-dependency or ICU settings with provision for ventilatory, cardiovascular, renal and other ancillary supports. MODS is potentially manageable when the factors responsible for the persistence or progression of MODS can be reversed. ${ }^{18}$

In our patient, release of adhesions at the first laparotomy, with subsequent reperfusion and exposure of PMNs to the previously ischemic, but viable gut vascular bed may have resulted in PMN priming by the same mechanism. The cascade of exaggerated responses as results of the second insults such as the intra-abdominal sepsis (bile peritonitis) and trauma of the second-look laparotomy resulted in MODS and mortality. The bile leak from the perforation of the gallbladder detected at the secondlook laparotomy could have resulted either from an injury during adhesiolysis at the initial laparotomy, or from an ischaemic infarction of the fundus of the gallbladder which has resulted from over-distension and pressure necrosis. One could speculate a missed perforation of the gallbladder at the first laparotomy, but the absence of free bile in the peritoneal cavity and lack of peritonism rule out this possibility. Meticulously performed surgery with attention to details and early recognition of intraabdominal catastrophe are paramount in the prevention of MODS.

\section{CONCLUSIONS}

In conclusion, despite progress in the ICU management of critically ill patients, MODS evolves and worsens, particularly in patients who undergo repetitive invasive procedures for infective conditions. Goal-directed therapies, supportive management, as well as an understanding of the inflammatory process are keys to decreasing the mortality rate among patients with MODS resulting from a second set of insults, which may be in the form of intra-abdominal sepsis, surgical trauma or a combination of both. 


\section{REFERENCES}

1. King JE. Sepsis in critical care. Crit Care Nurs Clin North Am 2007;19(1):77-86.

2. Saadia R, Schein M. Multiple organ failure. How valid is the "two hit" model? J Accid Emerg Med 1999;16(3):163-6; discussion 6-7.

3. Moore FA, Moore EE, Read RA. Postinjury multiple organ failure: role of extrathoracic injury and sepsis in adult respiratory distress syndrome. New Horiz 1993;1(4):538-49.

4. Keel M, Trentz O. Pathophysiology of polytrauma. Injury 2005;36(6):691-709.

5. Anderson BO, Harken AH. Multiple organ failure: inflammatory priming and activation sequences promote autologous tissue injury. J Trauma 1990;30(12 Suppl):S44-9.

6. Schaeffer V, Cuschieri J, Garcia I, et al. The priming effect of C5a on monocytes is predominantly mediated by the p38 MAPK pathway. Shock 2007;27(6):623-30.

7. Miyaoka K, Iwase M, Suzuki R, et al. Clinical evaluation of circulating interleukin- 6 and interleukin-10 levels after surgery-induced inflammation. J Surg Res 2005;125(2):14450 .

8. Bhatia M, Moochhala S. Role of inflammatory mediators in the pathophysiology of acute respiratory distress syndrome. J Pathol 2004;202(2):145-56

9. Zhang H, Slutsky AS, Vincent JL. Oxygen free radicals in
ARDS, septic shock and organ dysfunction. Intensive Care Med 2000;26(4):474-6

10. Krueger M, Heinzmann A, Nauck M. Adhesion molecules in pediatric intensive care patients with organ dysfunction syndrome. Intensive Care Med 2007;33(2):359-63.

11. Baue AE. Multiple organ failure, multiple organ dysfunction syndrome, and the systemic inflammatory response syndrome-where do we stand? Shock 1994;2(6):385-97.

12. Baue AE. MOF/MODS, SIRS: an update. Shock 1996;6 Suppl 1:51-5.

13. Baue AE. MOF, MODS, and SIRS: what is in a name or an acronym? Shock 2006;26(5):438-49.

14. Haga $\mathrm{Y}, \mathrm{Beppu} \mathrm{T}$, Doi K, et al. Systemic inflammatory response syndrome and organ dysfunction following gastrointestinal surgery. Crit Care Med 1997;25(12):1994-2000.

15. Cheatham ML. Intraabdominal pressure monitoring during fluid resuscitation. Curr Opin Crit Care. 2008 ;14(3):327-33

16. Maerz L, Kaplan LJ. Abdominal compartment syndrome. Crit Care Med. 2008 Apr;36(4 Suppl):S212-5.

17. Walsh CR. Multiple organ dysfunction syndrome after multiple trauma. Orthop Nurs 2005;24(5):324-33; quiz 34-5.

18. Schlichting D, McCollam JS. Recognizing and managing severe sepsis: a common and deadly threat. South Med J 2007;100(6):594-600. 